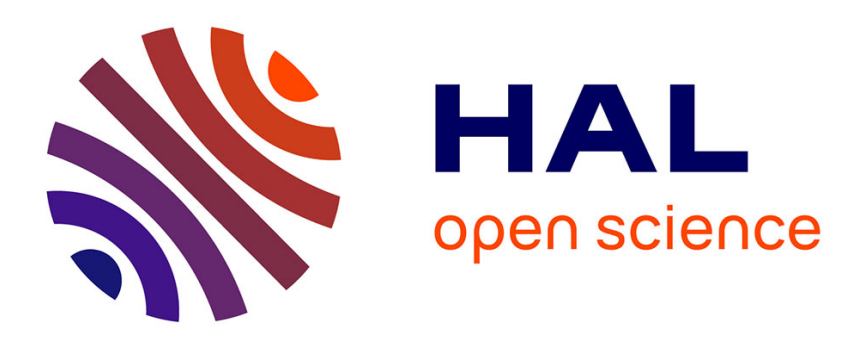

\title{
Une évaluation probabiliste pour l'erreur d'orientation des héliostats
}

\author{
V. Bădescu
}

\section{To cite this version:}

V. Bădescu. Une évaluation probabiliste pour l'erreur d'orientation des héliostats. Revue de Physique Appliquée, 1982, 17 (7), pp.421-434. 10.1051/rphysap:01982001707042100 . jpa-00245017

\section{HAL Id: jpa-00245017 https://hal.science/jpa-00245017}

Submitted on 1 Jan 1982

HAL is a multi-disciplinary open access archive for the deposit and dissemination of scientific research documents, whether they are published or not. The documents may come from teaching and research institutions in France or abroad, or from public or private research centers.
L'archive ouverte pluridisciplinaire HAL, est destinée au dépôt et à la diffusion de documents scientifiques de niveau recherche, publiés ou non, émanant des établissements d'enseignement et de recherche français ou étrangers, des laboratoires publics ou privés. 
Classification

Physics Abstracts

$86.10 \mathrm{~K}-86.30 \mathrm{~J}$

$86.30 S$

\title{
Une évaluation probabiliste pour l'erreur d'orientation des héliostats
}

\author{
V. Bădescu \\ I.F.A. Viscofil, Sos. Oltenitei 202, Bucharest, Roumanie
}

(Reçu le 17 mars 1981, révisé les 21 septembre 1981, ler février 1982, accepté le 14 avril 1982)

\begin{abstract}
Résumé. - Le travail étudie les erreurs d'orientation des systèmes concentrateurs formés d'un récepteur fixe et un, ou plusieurs héliostats. En utilisant certaines méthodes de la géométrie intégrale on a établi les expressions de ces erreurs au cas où l'orientation des héliostats est contrôlée par des senseurs. On montre que par la décentralisation du senseur il est possible de diminuer l'erreur moyenne quotidienne. On indique la modalité par laquelle la méthode peut être utilisée pour d'autres systèmes de commande de l'orientation. On discute la validité des distributions des erreurs d'orientation proposées dans la littérature. Les relations obtenues peuvent être utilisées directement dans les modèles de calcul de l'intensité de la radiation solaire concentrée pour le cas des systèmes aux champs d'héliostats.
\end{abstract}

\begin{abstract}
The paper studies the tracking errors of the concentrating systems formed of heliostats and a fixed receiver. Using methods of the integral geometry, it has been established the expressions of these errors in the case that the heliostats orientation is controlled by a sensory system. It has already been shown that by the decentralization of the sensory it is possible to diminish the dayly average error. It is stated the modality in which the method can be used for other control systems than the sensory ones. It is discussed the correctness of the tracking errors distributions proposed in the literature. The resulting relations can be used directly in the models of solar flux distribution in the case of systems with heliostats fields.
\end{abstract}

1. Introduction. - La théorie mathématique du processus de concentration de la radiation solaire à l'aide des héliostats a fait l'objet de nombreux travaux, parmi lesquels nous citerons seulement $[1,2,3,4,5,6]$. Par conséquent, on a élaboré des techniques spécifiques pour le calcul de la distribution de l'intensité de la radiation solaire concentrée, pour des miroirs singuliers $[1,2,3]$ ainsi que pour des champs d'héliostats $[4,5,6]$. Du point de vue de la technique mathématique utilisée, il y a deux méthodes principales de calcul [7]. Une première méthode, moins précise, mais plus manouvrable, est la méthode de l'approximation gaussienne. Dans ce cas, en utilisant l'équation Aparisi on détermine le flux de la radiation solaire concentrée dans le plan focal, sous la forme d'une distribution gaussienne [8]. La seconde méthode, plus récente, utilise comme instrument de travail des programmes complets de calcul (ray tracing programs), à l'aide desquels elle essaye de tenir compte de tous les paramètres physiques du phénomène optique étudié.

Pour que ces techniques, analytiques ou algorithmiques, constituent une bonne modélisation du fonc- tionnement réel des systèmes concentrateurs, il a été nécessaire d'introduire quelques corrections par lesquelles on puisse tenir compte également de l'existence de certains phénomènes perturbateurs. Les plus souvent rappelées dans la littérature sont les erreurs qui peuvent affecter la qualité et le contour de la surface réfléchissante et les erreurs d'orientation des héliostats. Les modalités par lesquelles ces deux types d'erreurs sont introduits dans le calcul sont très différentes. Ainsi, pour des champs d'héliostats, Riaz et Gurr [4] introduisent un facteur global $k$ de dispersion du faisceau réfléchi, qui prend en considération les erreurs de surface et d'orientation du miroir réfléchissant. Igel et Hughes [9], rappellent ces deux catégories d'erreurs, mais introduisent un facteur correcteur qui tient compte seulement des déformations de la surface des miroirs. De plus, McFee [6] admet dans ses calculs des distributions gaussiennes de dispersion connue pour les erreurs de surface, ainsi que pour celles d'orientation. La même hypothèse avait été utilisée antérieurement par Umarov [8]. Lipps et Walzel [10], supposent que toutes les catégories de perturbations de la réflexion peuvent être décrites par une seule 
distribution, formée d'un polynôme de fonctions trigonométriques, dont les coefficients sont déterminés empiriquement. Dans un travail récent, Ittner III [11] ne considère pas connue la distribution des erreurs d'orientation, mais seulement l'erreur maximale possible. Il étudie l'influence que cette erreur maximale a sur l'étendue de la zone du plan focal où l'intensité de la radiation solaire concentrée est uniforme. En même temps, dans [11] on précise que l'ignorance de la distribution des erreurs d'orientation a comme effet l'impossibilité de déterminer correctement l'intensité de la radiation solaire concentrée, dans la zone du plan focal où cette dernière a une distribution non uniforme. L'étude la plus complète concernant l'influence que les erreurs d'orientation ont sur la distribution du flux de la radiation concentrée est effectuée par Hughes $[7,12,13]$. Dans ses travaux il part de la prémisse d'une distribution uniforme des erreurs d'orientation dans une certaine zone, supposée connue, du plan focal. On tire la conclusion que, grâce à leur spécificité, les erreurs d'orientation doivent être traitées séparément de celles qui considèrent la qualité de la surface réfléchissante.

Les techniques de calcul citées, quoique très différentes, se ressemblent du fait que, d'une façon ou d'une autre, elles supposent connues soit les deux distributions des erreurs, soit certaines grandeurs qui peuvent en résulter par le traitement de ces dernières. Mais, en même temps on observe que la manière dont est choisie la forme des distributions des erreurs est différente selon l'auteur et qu'elle n'est pas toujours suffisamment argumentée. Cette situation exige des études systématiques précises pour déterminer l'allure des deux types principaux de distributions des erreurs. Une tentative de détermination théorique de la distribution des erreurs d'orientation a été faite par Bădescu [14], qui a utilisé des méthodes de la géométrie intégrale. Le modèle physique développé en [14] est spécifique pour les systèmes concentrateurs formés d'un récepteur fixe et un, ou plusieurs, héliostats actionnés indépendamment, où l'orientation est assurée par un senseur. Ce modèle permet l'évaluation de l'erreur d'orientation pour chaque miroir, sans tenir compte du fait que la surface réfléchissante est plane, ou courbée. Dans le travail présent nous nous proposons d'étendre et de systématiser la méthode de calcul présentée en [14] et de montrer la modalité de généraliser cette méthode pour le cas des systèmes de concentration pour lesquels le contrôle de l'orientation est assuré par d'autres moyens que par l'intermédiaire d'un senseur.

2. Le processus d'orientation des héliostats. Le processus d'orientation des héliostats est réalisé par l'intermédiaire de deux systèmes : un système de commande de l'orientation et un système d'actionnement. Le premier système a le rôle d'établir la direction, le sens et la grandeur par laquelle l'inclinaison du miroir doit être corrigée, tandis que le second système assure le mouvement de l'héliostat. Ensuite, on suppose que la fonction de commande de l'orientation, est accomplie à l'aide d'un senseur. En même temps, aucune hypothèse restrictive ne sera faite en ce qui concerne la nature du système d'actionnement.

2.1 Notions ET DÉFINITIONS PRÉLIMINAIRES. Nous noterons : par $\mathrm{O}_{1}$ (Fig. 1) le centre de mouvement de l'héliostat, c'est-à-dire le point idéal et fixe autour duquel se tourne la surface réfléchissante pendant une étape de fonctionnement dépourvue de l'intervention des forces mécaniques perturbatrices.

Par $\mathrm{O}_{1}^{*}$ le centre, généralement mobile, de la surface réfléchissante. Dans le cas idéal les deux centres devraient coïncider. Dans la pratique, le point $\mathrm{O}_{1}$ peut être différent de $\mathrm{O}_{1}^{*}$, parfois pouvant être extérieur à la surface réfléchissante du miroir.

$\mathrm{Par} \mathrm{O}_{3}$ le centre optique du système (le foyer). Nous définirons l'axe optique du système, par la droite fixe qui unit les points $\mathrm{O}_{1}$ et $\mathrm{O}_{3}$. Nous dénommerons plan image, $(\Pi)$, le plan passant par $\mathrm{O}_{3}$ et perpendiculaire à l'axe optique.

$\mathrm{Par} \mathrm{O}_{2}$ le point d'intersection de l'axe optique avec la surface de disposition des éléments opto-électroniques du senseur d'orientation. Nous supposerons que le senseur est sensible à la direction de réflexion du rayon solaire, incident en $\mathrm{O}_{1}^{*}$. Nous appellerons erreurs d'orientation, les déviations par rapport à l'axe optique du rayon réfléchi de $\mathrm{O}_{1}^{*}$, qui sont dues aux imprécisions dans la réalisation de l'inclinaison de l'héliostat, dans l'hypothèse d'une qualité parfaite de la surface réfléchissante. Nous préfèrerons cette dénomination par rapport aux autres expressions utilisées dans la littérature (voir, steering errors [4], guidance errors [5], tracking errors [6]). Nous noterons par $\bar{I}$ et nous dénommerons rayon incident principal, le rayon solaire incident au point $\mathrm{O}_{1}^{*}$. Nous noterons par $\bar{R}$, et nous dénommerons rayon réfléchi principal, le rayon solaire réfléchi du point $\mathrm{O}_{1}^{*}$. L'ensemble des notations adoptées est présente dans la figure 1 , pour le cas idéal où $\mathrm{O}_{1}^{*} \equiv \mathrm{O}_{1}$.

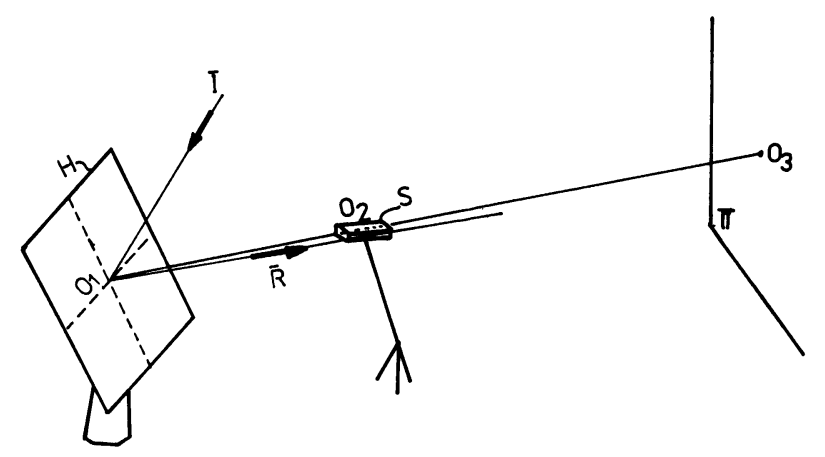

Fig. 1. - Système concentrateur de radiation solaire, formé d'un héliostat et d'un récepteur fixe $\left(\mathrm{O}_{1} \equiv \mathrm{O}_{1}^{*}\right)$.

[System of solar energy concentration, formed of a heliostat and a fixed receiver $\left(\mathrm{O}_{1} \equiv \mathrm{O}_{1}^{*}\right)$.] 
2.2 LA FONCTION DU SENSEUR D'ORIENTATION. Nous supposerons pour le début que le rayon réfléchi principal a la direction de l'axe optique. Dans ce contexte, le senseur ne commandera pas le mouvement de correction de l'orientation de l'héliostat. Bien que l'inclinaison du miroir restera ensuite invariable, à cause du mouvement apparent du Soleil sur la voûte céleste, le rayon réfléchi principal changera graduellement sa direction, déviant de la ligne de l'axe optique.

Pour que le rayon réfléchi principal, coïncide de nouveau avec la direction de l'axe optique, il est nécessaire de corriger, en permanence, l'orientation de l'héliostat. En principe, les senseurs d'orientation des héliostats sont conçus de sorte que le faisceau de rayons réfléchis qui pénètre à l'intérieur de la lunette de visée peut rencontrer deux régions. L'une des régions (la zone insensible), localisée sur la direction de l'axe optique $\Gamma$ (la zone $\mathrm{S}_{2}^{\prime}$ de la figure 2), ne réagit pas à la réception de l'énergie du faisceau de rayons solaires. L'autre région (la zone sensible), qui entoure la zone $S_{2}^{\prime}$, contient des dispositifs opto-électroniques qui ont le rôle de détecter la direction et la grandeur de la déviation des rayons solaires réfléchis, par rapport à l'axe optique. Au moment où on atteint une déviation critique, les rayons de 'extrémité du faisceau lumineux quittent la zone $S_{2}^{\prime}$ et pénètrent dans la zone de sensibilité. Par conséquent, un signal d'erreur est émis. Après amplification, ce signal est appliqué aux actionneurs qui corrigent l'orientation de l'héliostat, de sorte que le faisceau lumineux revienne à l'intérieur de la zone insensible [15]. La condition de déclenchement du signal d'erreur est équivalente avec la sortie du rayon réfléchi principal (situé au centre du faisceau lumineux qui pénètre dans la lunette de visée) de l'intérieur d'une zone $S_{2}$, incluse dans la zone insensible $S_{2}^{\prime}$. Nous appellerons la zone $S_{2}$, zone insensible réduite. On observe que dans le cas idéal où le faisceau lumineux qui pénètre dans la lunette de visée se réduit au rayon réfléchi principal, les zones $S_{2}$ et $S_{2}^{\prime}$ coïncident.

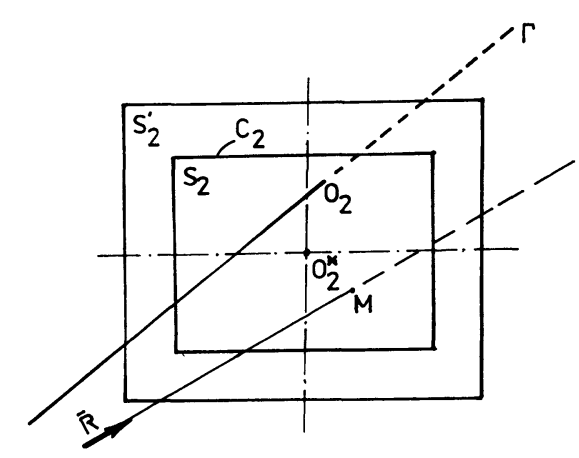

Fig. 2. - Zone insensible $S_{2}^{\prime}$ et zone insensible réduite $S_{2}$ d'un senseur non centré. $\Gamma$-axe optique, $\bar{R}$-rayon réfléchi principal.

[The insensible zone $S_{2}^{\prime}$ and the reduced insensible zone $S_{2}$ of an eccentric sensory system. $\Gamma$-optic axis, $\bar{R}$-main reflected ray.]
Nous noterons par $\mathrm{O}_{2}^{*}$ le centre de la zone d'insensibilité réduite. Un montage correct du senseur exige le placement de $\mathrm{O}_{2}^{*}$ sur l'axe optique du miroir. La position de $\mathrm{O}_{2}^{*}$ par rapport à l'axe optique classifie les senseurs en senseurs centrés $\left(\mathrm{O}_{1} \mathrm{O}_{2}^{*} \equiv \mathrm{O}_{1} \mathrm{O}_{3}\right)$ et non centrés $\left(\mathrm{O}_{1} \mathrm{O}_{2}^{*} \neq \mathrm{O}_{1} \mathrm{O}_{3}\right)$. On observe que dans le cas des senseurs centrés, $\mathrm{O}_{2}^{*} \equiv \mathrm{O}_{2}$. La zone insensible réduite de la figure 2 est caractéristique pour un senseur non centré.

Projetée du point $O_{1}$ au niveau du plan image, la zone insensible réduite détermine une zone $\mathrm{S}_{3}$, délimitée par la courbe $\mathrm{C}_{3}$ (voir Annexe). Tant que la condition d'orientation est accomplie, le rayon réfléchi principal croisera le plan image à l'intérieur de la zone $S_{3}$. Sur la figure 3 est présentée la construction graphique de la zone $S_{3}$, en utilisant pour cela la zone insensible réduite supposée connue, d'un senseur centré.

En pratique, le point $\mathrm{O}_{1}^{*}$ est différent du centre idéal de mouvement $\mathrm{O}_{1}$; ceci due à la construction de l'héliostat, qui ne permet pas au centre $\mathrm{O}_{1}^{*}$ de la surface réfléchissante de rester fixe pendant le fonctionnement. Au cas où cette condition est accomplie, il est pourtant possible que le point $\mathrm{O}_{1}^{*}$ ne soit pas fixe, à cause des phénomènes perturbateurs qui peuvent apparaitre pendant le fonctionnement quotidien.

Considérons le cas général, nous supposerons que le point mobile $\mathrm{O}_{1}^{*}$ décrit, dans une période de temps donnée, une surface $S_{1}$ confinée avec une courbe $C_{1}$ (Fig. 4). A cause du fait qu'à un certain moment le

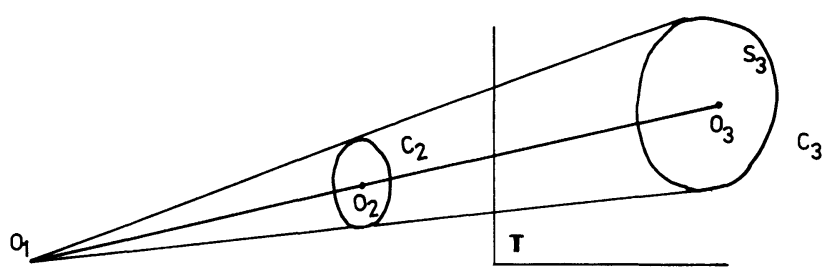

Fig. 3. - Construction de la zone $S_{3}$ au cas où $O_{1}^{*} \equiv O_{1}$ et $\mathrm{O}_{2} \equiv \mathrm{O}_{2}^{*}$.

[The construction of the zone $S_{3}$ in the case when $O_{1}^{*} \equiv O_{1}$ and $\mathrm{O}_{2} \equiv \mathrm{O}_{2}^{*}$.]

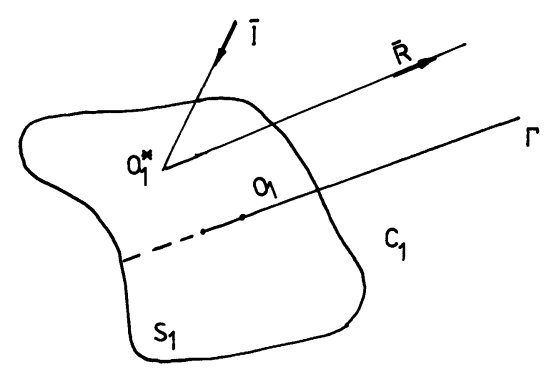

Fig. 4. - La zone $S_{1}$ décrite par le centre $O_{1}^{*}$ mobile de la surface de l'héliostat. $\Gamma$-axe optique, $\bar{I}$-rayon incident principal, $\bar{R}$-rayon réfléchi principal.

[The region $S_{1}$ described by the unstable centre $O_{1}^{*}$ of the heliostat surface. $\Gamma$-optic axis, $\bar{I}$-main incident ray, $R$-main reflected ray.] 
point $\mathrm{O}_{1}^{*}$ ne coïncide pas avec le point $\mathrm{O}_{1}$, et que sa position n'est pas absolument connue, la manière de déterminer la zone $S_{3}$ sera modifiée. En effet, chaque point de $S_{1}$ étant un point possible d'origine du rayon réfléchi principal, la projection de la zone insensible au niveau du plan image ne sera plus unique. Le procédé de détermination de la zone $S_{3}^{*}$, qui remplacera la zone $S_{3}$, est le suivant (voir Annexe) :

a) Pour chaque point $\mathrm{M}$ de la courbe $\mathrm{C}_{1}$ on construit un cône $\mathrm{K}_{\mathrm{M}}$, de sommet $\mathrm{M}$, dont les génératrices s'appuient sur la courbe $C_{2}$. L'ensemble de ces cônes sera noté par $\left\{\mathrm{K}_{\mathrm{M}}\right\}$.

b) On construit l'enveloppe $\mathrm{E}_{\mathrm{K}}$ de l'ensemble des cônes $\left\{\mathrm{K}_{\mathrm{M}}\right\}$.

c) L'intersection de la surface $E_{K}$, avec le plan image, déterminera la courbe $\mathrm{C}_{3}^{*}$, qui confine la zone $\mathrm{S}_{3}^{*}$.

Donc, on observe que, à cause de l'instạbilité du centre du miroir réfléchissant, tant que la condition d'orientation est accomplie, le rayon réfléchi principal rencontrera le plan image dans une zone $S_{3}^{*}$, plus étendue que la zone $S_{3}$.

2.3 LES FACTEURS ALÉATOIRES IMPLIQUÉS DANS L'ORIENTATION DES HÉLIOSTATS. - On considère que les zones $S_{1}$ et $S_{2}$, supposées connues, ont déterminé, en utilisant le procédé décrit en $\S 2.2$, une zone $\mathrm{S}_{3}^{*}$ de la forme présentée sur la figure 5 . Au début, on suppose que le rayon réfléchi principal rencontre le plan image au point $\mathrm{A}$. Comme résultat de la commande d'orientation, le rayon réfléchi principal parcourt la distance $\mathrm{AB}$, touchant la frontière de la zone $\mathrm{S}_{3}^{*}$. La conséquence de ce fait, qui correspond à l'entrée du rayon réfléchi dans la zone insensible réduite du senseur, est que la commande d'orientation s'arrête. Mais à cause des inerties, et à cause de certaines influences perturbatrices possibles, le rayon réfléchi principal pénètre sur une distance inconnue $\mathrm{BC}$ à l'intérieur de la zone $S_{3}^{*}$. Dans la période suivante l'héliostat étant orienté, restera immobile. Cependant, le rayon réfléchi principal parcourra la distance $\mathrm{CD}$, comme résultat du mouvement apparent du Soleil sur la voûte

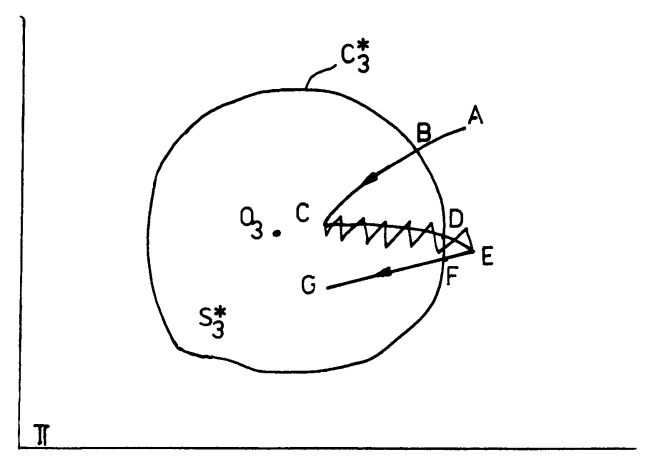

Fig. 5. - Trajectoire du point d'intersection du rayon réfléchi principal avec le plan image $\Pi . \mathrm{O}_{3}$-centre optique.

[The displacement of the intersection point of the main reflected ray with the image plane $\Pi . \mathrm{O}_{3}$-optic centre.] céleste. Au moment où il quitte, sur une très courte distance DE, la zone $\mathrm{S}_{3}^{*}$, le senseur. commande la correction de l'orientation. Cette commande s'arrête, au moment de la pénétration du rayon réfléchi principal à l'intérieur de la zone $S_{3}^{*}$ (le point $F$ ) mais, à cause des inerties mentionnées le mouvement continue encore sur la distance FG. Le processus discontinu de correction de l'orientation se déroule ensuite selon le mécanisme décrit ci-dessus. Cette description simplifiée ne tient pas compte de l'effet de tous les facteurs perturbateurs, qui peuvent influencer l'orientation des héliostats. Aussi, une description plus réaliste du tracé du rayon réfléchi principal sera donnée par la ligne brisée, superposée sur le segment de courbe CD (Fig. 5).

Le temps où le rayon réfléchi principal se trouve en dehors de la zone $S_{3}^{*}$ peut être diminué autant que l'on veut par rapport au temps où il se trouve à l'intérieur de cette zone, par un choix convenable de la sensibilité des éléments opto-électroniques. Par conséquent, nous supposerons, avec une très bonne approximation, que durant le fonctionnement de l'héliostat, le rayon réfléchi principal croise en permanence le plan image à l'intérieur de la zone $\mathrm{S}_{3}^{*}$.

L'analyse présentée souligne le fait qu'on peut connaître avec précision dans quelle zone du plan image se réfléchira le rayon incident principal, et qu'il y a une méthode précise pour déterminer cette zone. Mais nous pouvons savoir avec un certain degré de précision en quel point de la zone $\mathrm{S}_{3}^{*}\left(\right.$ ou $\mathrm{S}_{3}$ ) se réfléchit le rayon incident principal. C'est pourquoi nous appellerons la zone $\mathrm{S}_{3}^{*}\left(\right.$ ou $\left.\mathrm{S}_{3}\right)$ zone d'imprécision de la réflexion du rayon incident principal.

L'imprécision qui caractérise le processus d'orientation est le résultat de l'action de trois groupes de facteurs aléatoires.

Un premier groupe caractérise la localisation du centre $\mathrm{O}_{1}^{*}$ de l'héliostat. Le placement de ce point est très important, parce qu'il détermine le centre de l'image réfléchie du miroir dans le plan $\Pi$. Bien que dans les calculs analytiques $[4,5,9,10]$ on considère que le centre $\mathrm{O}_{1}^{*}$ du miroir ait une position déterminée, spécifiée par le point fixe $\mathrm{O}_{1}$, dans la pratique il peut supporter des déviations par rapport à ce point idéal. Les causes qui déterminent le plus souvent ces déviations sont : les solutions défectueuses de construction, les rafales de vent, les dilatations thermiques différentielles des composantes de l'héliostat [16].

Un deuxième groupe de facteurs aléatoires intervient au niveau de la localisation du senseur. Dans le cas idéal le senseur peut être considéré centré sur l'axe optique. Mais, en pratique il est plus ou moins non centré (erreurs de réglage apparues au cours du montage ou au cours des opérations d'entretien périodique).

Le troisième groupe de facteurs aléatoires apparaît au cours de l'orientation des héliostats. La cause perturbatrice la plus souvent rencontrée est l'inertie du système d'actionnement. 
3. Le calcul de la distribution des erreurs d'orientation. - Nous choisirons un système orthogonal de coordonnées, ayant l'origine en $\mathrm{O}_{1}$, l'axe $\mathrm{O}_{1} z$ passant par le centre optique $\mathrm{O}_{3}$ (Fig. 6), l'axe $\mathrm{O}_{1} y$ parallèle à la surface du sol. L'intersection de l'enveloppe $\mathrm{E}_{\mathrm{K}}$ déterminée en $\S 2.2$, avec le plan $\mathrm{O}_{1} x y$ déterminera une courbe $C_{1}^{*}$, qui confine à une région fermée $S_{1}^{*}$ de ce plan (voir Annexe). Quel que soit le point de réflexion $\mathrm{O}_{1}^{*}$ appartenant à la région $\mathrm{S}_{1}$, le rayon réfléchi principal passera par la surface $S_{1}^{*}$. Nous noterons $\mathrm{O}^{*}$ son intersection avec le plan $\mathrm{O}_{1} x y$. On observe que le même rayon réfléchi principal peut être précisé en connaissant soit la position du point d'origine $\mathrm{O}_{1}^{*}$, soit la position de la projection $\mathrm{O}^{*}$ de ce point dans le plan $\mathrm{O}_{1} x y$. Il est convenable de suivre la seconde variante, pour simplifier les calculs ultérieurs. En effet, on observe que dans le premier cas le domaine de variation du point $O_{1}^{*}$ est la région $S_{1}$, généralement non plane, tandis que dans le second cas le domaine de variation du point $\mathrm{O}^{*}$ est la région plane $S_{1}^{*}$. Tout rayon réfléchi principal est défini dans le système $\mathrm{O}_{1} x y z$ par les équations d'une droite :

$$
\text { (4) } \begin{aligned}
& x=a z+u \\
& y=b z+v
\end{aligned}
$$

où $a, b, u, v$, sont des paramètres spécifiques.

Le processus d'orientation de l'héliostat fait que l'origine et la direction du rayon réfléchi principal varie de façon permanente tout au long de la journée. Au niveau de la représentation (1) du rayon réfléchi principal, ceci se manifeste par la variation des paramètres $a, b, u, v$. Bien que ces paramètres varient d'une manière aléatoire, leurs domaines de définition sont connus par le fait que le rayon réfléchi principal ne peut pas sortir hors du volume confiné à la surface $\mathrm{E}_{\mathrm{K}}$.

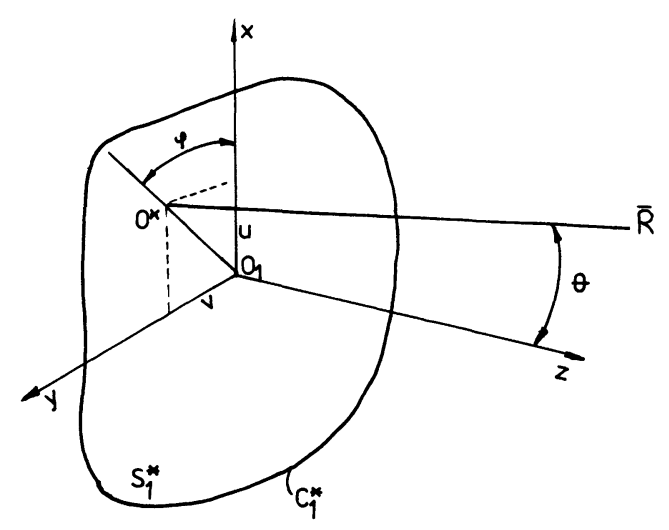

Fig. 6. - La zone $S_{1}^{*}$ et le point $O^{*}$, l'équivalent dans le plan $\mathrm{O}_{1} x y \mathrm{du}$ point $\mathrm{O}_{1}^{*}$. $\mathrm{O}_{1} z$-axe optique, $\bar{R}$-rayon réfléchi principal ; $(\varphi, \theta)$-angles caractérisant l'inclinaison du rayon $\bar{R}$ par rapport à $\mathrm{O}_{1} z$.

[The region $\mathrm{S}_{1}^{*}$ and the point $\mathrm{O}^{*}$, the equivalent in the $\mathrm{O}_{1} x y$ plane of the point $\mathrm{O}_{1}^{*}$. $\mathrm{O}_{1} z$-optic axis, $\bar{R}$-main reflected ray ; $\varphi, \theta$-angles characterizing the inclination of the ray $\bar{R}$ in comparison with $\mathrm{O}_{1} z$.]
En même temps quel que soit le mode de variation de $a, b, u, v$, la forme (1) reste invariable pendant la journée. Nous noterons par (D) l'ensemble des droites de la forme (1). Parce que chaque rayon réfléchi principal est univoquement déterminé par une valeur établie des paramètres $a, b, u, v$, l'ensemble (D) sera univoquement déterminé, par les domaines de variation de ces quatre paramètres. Il en résulte que le processus d'orientation de l'héliostat transforme des droites de l'ensemble (D) en droites du même ensemble. $\mathrm{Du}$ point de vue mathématique cela signifie que le processus déjà mentionné laisse invariant l'ensemble (D) des droites. On sait $[17,18]$ que l'ensemble des transformations géométriques qui laissent invariant l'ensemble (D) est constitué par le groupe $H_{T}$ des transformations orthogonales de l'espace réel $\mathbf{R}_{\mathbf{3}}$. Comme conséquence, ce groupe de transformations est l'équivalent géométrique du processus d'orientation du miroir.

A cause des perturbations mentionnées dans le paragraphe 2.3, il est impossible de connaître précisément la position du rayon réfléchi principal. Mais, les hypothèses acceptées jusqu'ici nous permettent d'affirmer avec certitude le fait que le rayon réfléchi principal appartient à l'ensemble (D) des droites de la forme (1), qui ont l'origine dans la zone $S_{1}^{*}$ et qui croisent la zone d'imprécision $\mathrm{S}_{3}^{*}$. Nous nous proposons de déterminer la probabilité pour que le rayon réfléchi principal appartienne au sous-ensemble (S) des droites, supposé connu, inclus dans l'ensemble (D). De même que l'ensemble (D) a été précisé par un domaine [D] de l'espace $\mathrm{P}_{4}$ des paramètres $(a, b, u, v)$, nous préciserons aussi le sous-ensemble (S) comme étant déterminé par un domaine $[S] \subset[D]$ du même espace $\mathbf{P}_{4}$.

Il ést normal de supposer que la probabilité recherchée est d'autant plus grande que la mesure du sousensemble (S) est elle aussi plus grande. Au cas où le sous-ensemble (S) coïnciderait avec l'ensemble (D), la probabilité devrait être égale à l'unité. Ainsi, nous arrivons naturellement à la définition donnée en probabilitées géométriques $[17,18]$ :

$$
P(\mathrm{~S})=\frac{\mu(\mathrm{S})}{\mu(\mathrm{D})}
$$

où $\mu(\mathrm{S}), \mu(\mathrm{D})$ désignent les mesures de l'ensemble (S), et de l'ensemble (D).

Un résultat classique en géométrie intégrale $[17,18]$ montre que la mesure d'un ensemble de figures géométriques $(\mathrm{F})$, invariant à l'action d'un certain groupe de transformations géométriques $\mathrm{H}$, est donnée par :

$$
\mu(\mathrm{F})=\int_{[\mathrm{F}]} \cdots \int \phi \mathrm{d} \Omega .
$$

En (3), $\phi$ est la fonction invariante intégrale du groupe de transformations $\mathrm{H}, \Omega$ est le volume de l'espace des paramètres qui définissent l'ensemble $(F)$, et $[F]$ est 
le domaine de variation de ces paramètres. Dans le cas étudié, la famille (F) de figures géométriques doit être identifié avec l'ensemble (D). Il est aussi facile d'observer que le groupe $\mathbf{H}$ doit être identifié avec le groupe $\mathbf{H}_{\mathrm{T}}$ des transformations orthogonales, qui maintient invariant l'ensemble (D). Pour calculer la mesure (3) il est donc nécessaire, de connaître la fonction invariante intégrale du groupe de transformations $\mathrm{H}_{T}$. Cette fonction peut être déterminée en solutionnant le système du Deltheil d'équations différentielles $[17,18]$ :

$$
\begin{gathered}
b \frac{\partial \phi}{\partial a}-a \frac{\partial \phi}{\partial b}+v \frac{\partial \phi}{\partial u}-u \frac{\partial \phi}{\partial v}=0 \\
a b \frac{\partial \phi}{\partial a}+\left(1+b^{2}\right) \frac{\partial \phi}{\partial b}+a v \frac{\partial \phi}{\partial u}+b v \frac{\partial \phi}{\partial v}=-4 b \phi \\
\left(1+a^{2}\right) \frac{\partial \phi}{\partial a}+a b \frac{\partial \phi}{\partial b}+a u \frac{\partial \phi}{\partial u}+b u \frac{\partial \phi}{\partial v}=-4 a \phi \\
\frac{\partial \phi}{\partial u}=0 \\
\frac{\partial \phi}{\partial v}=0 \\
a \frac{\partial \phi}{\partial u}+b \frac{\partial \phi}{\partial v}=0
\end{gathered}
$$

La solution du système (4) est la suivante $[17,18]$ :

$$
\phi(a, b, u, v)=\left(a^{2}+b^{2}+1\right)^{-2} .
$$

Au cas où la monture de l'héliostat est très rigide, et le centre $O_{1}^{*}$ de la surface réfléchissante coïncide avec le centre de mouvement $\mathrm{O}_{1}$, on peut considérer que la surface de la zone $S_{1}$ a une mesure nulle. Dans ce cas, tous les rayons réfléchis principaux possibles auront leur origine en $\mathrm{O}_{1}^{*} \equiv \mathrm{O}_{1}$, et se différencieront seulement par l'inclinaison par rapport à l'axe optique. C'est pourquoi, le nombre des paramètres aléatoires qui caractérisent le processus d'orientation se réduit à deux $(a$ et $b$ ). Dans cette situation le système du Deltheil d'équations différentielles aura la forme [17] :

$$
\begin{gathered}
b \frac{\partial \phi}{\partial a}-a \frac{\partial \phi}{\partial b}=0 \\
a b \frac{\partial \phi}{\partial a}+\left(1+b^{2}\right) \frac{\partial \phi}{\partial b}=-3 b \phi \\
\left(1+a^{2}\right) \frac{\partial \phi}{\partial a}+a b \frac{\partial \phi}{\partial b}=-3 a \phi .
\end{gathered}
$$

La solution du système (6) est [17] :

$$
\phi(a, b)=\left(a^{2}+b^{2}+1\right)^{-3 / 2} .
$$

Dans les conditions du cas général, en utilisant la relation (2), on déterminera la probabilité $P(\mathrm{~S})$ que le rayon réfléchi principal appartienne à un sousensemble (S) de droites. On observe immédiatement que, quelque soit le sous-ensemble (S), le dénominateur de l'expression (2) aura toujours la même valeur. Donc, tenant compte de (3), la probabilité cherchée sera donnée par :

$$
P(\mathbf{S})=C \iiint \int_{[S]} \phi(a, b, u, v) \mathrm{d} a \mathrm{~d} b \mathrm{~d} u \mathrm{~d} v
$$

où $C$ est une constante de normalisation. L'expression (8) permet de déterminer la densité de probabilité $p(a, b, u, v)$ :

$$
p(a, b, u, v)=C \phi(a, b, u, v) .
$$

La constante de normalisation est déterminée en utilisant la condition :

$$
\iiint \int_{[\mathrm{D}]} p \mathrm{~d} a \mathrm{~d} b \mathrm{~d} u \mathrm{~d} v=1
$$

On observe donc que la valeur acquise par la fonction invariante intégrale en un point quelconque $\mathrm{M}(a, b, u, v)$ de l'espace $\mathrm{P}_{4}$, est à une constante près, la probabilité de réalisation du rayon réfléchi principal univoquement déterminé par le point $\mathbf{M}$. Cela prouve le rôle prépondérant que la fonction $\phi$ a pour la détermination des erreurs d'orientation.

Dans les deux paragraphes suivants, on utilisera la relation (9) pour déterminer la distribution des erreurs d'orientation. Cette distribution sera exprimée sous deux formes différentes, pour permettre son utilisation dans les modèles de calcul de l'intensité de la radiation solaire concentrée qui tiennent compte de l'erreur angulaire de déviation du rayon réfléchi principal par rapport à l'axe optique [5, 10], ainsi que dans les modèles qui tiennent compte de la distance qui sépare le point d'intersection du rayon réfléchi principal avec le plan image, par rapport au centre optique $[6,7]$.

3.1 L'ERREUR D'ORIENTATION DES HÉLIOSTATS POUR LESQUELS LE CENTRE O* EST FIXE. - Nous avons montré qu'au cas où le centre $\mathrm{O}_{1}^{*}$ des héliostats est fixe pendant le fonctionnement, il doit coïncider avec le centre de mouvement $\mathrm{O}_{1}$. Dans ce cas, le nombre des paramètres aléatoires se réduit à deux, la zone d'imprécision est $S_{3}$ et l'expression (7) reste valable. La densité de probabilité aura donc l'expression :

$$
p(a, b)=C \phi(a, b)=C\left(a^{2}+b^{2}+1\right)^{-\frac{3}{2}} .
$$

Pour donner un sens physique précis à l'expression (11) nous tiendrons compte du fait que, dans le cas étudié, tous les rayons réfléchis principaux ont le même point d'origine $\mathrm{O}_{1}$ et se différencient seulement par l'angle de déviation par rapport à l'axe optique. Nous noterons avec $\theta$ l'angle formé par un rayon réfléchi principal quelconque $\bar{R}$ avec l'axe optique $\mathrm{O}_{1} z$ et avec $\varphi$ l'angle formé par la projection de $R$ sur le plan $\mathrm{O}_{1} x y$ avec 
l'axe $\mathrm{O}_{1} x$ (Fig. 7). On observe de (1) et de la figure 7 que :

$$
\begin{aligned}
& a=\cos \varphi \operatorname{tg} \theta \\
& b=\sin \varphi \operatorname{tg} \theta .
\end{aligned}
$$

Le changement de variables (12) a le jacobien :

$$
\frac{D(a, b)}{D(\theta, \varphi)}=-\frac{\sin \theta}{\cos ^{3} \theta}
$$

Remplaçant (11), (12), (13) en (8) nous trouvons :

$$
P(\mathbf{S})=C \iint_{[\mathrm{S}]_{\mathbf{T}}}|\sin \theta| \mathrm{d} \varphi \mathrm{d} \theta
$$

où $[\mathrm{S}]_{\mathrm{T}}$ est le sous-domaine de variation des paramètres $(\theta, \varphi)$ correspondant par l'intermédiaire des transformations (12) au domaine [S]. Il en résulte que la fonction invariante intégrale a, dans les nouvelles coordonnées, l'expression :

$$
\phi(\theta, \varphi)=|\sin \theta|
$$

et la densité de probabilité des erreurs d'orientation $(\theta, \varphi)$ sera :

$$
p(\theta, \varphi)=C|\sin \theta|
$$

La détermination de la constante $C$ est faite par la condition usuelle :

$$
\iint_{[D]_{\mathbf{T}}} p(\theta, \varphi) \mathrm{d} \theta \mathrm{d} \varphi=1
$$

où $[D]_{T}$ est le domaine entier d'intégration des paramètres $(\theta, \varphi)$.

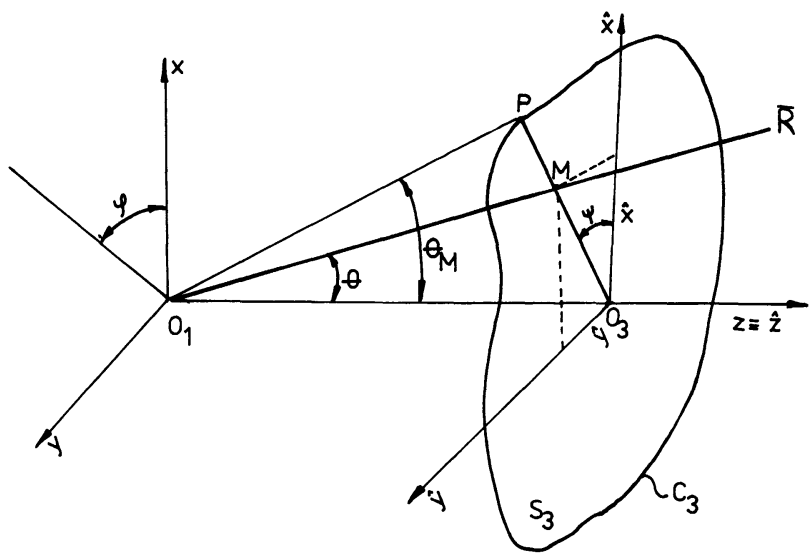

Fig. 7. - Les paramètres impliqués dans le calcul de la distribution des erreurs d'orientation $\left(\mathrm{O}_{1}^{*} \equiv \mathrm{O}_{1}\right) . \mathrm{O}_{1} \mathrm{O}_{3}$-axe optique, $(\varphi, \theta, \psi)$-angles caractérisant l'inclinaison de $\bar{R}$ par rapport à $\mathrm{O}_{1} \mathrm{O}_{3}$.

[The parameters involved in the calculus of the steering errors distribution $\left(\mathrm{O}_{1}^{*} \equiv \mathrm{O}_{1}\right) . \mathrm{O}_{1} \mathrm{O}_{3}$-optic axis $; \varphi, \theta, \psi$ angles characterizing the inclinaison of the ray $\bar{R}$ in comparison with $\mathrm{O}_{1} \mathrm{O}_{3}$.]
Donnons la manière de déterminer le domaine $[D]_{T}$, qui suppose connue la zone d'imprécision $\mathrm{S}_{3}$. Considérons que le rayon réfléchi principal $\bar{R}$ rencontre le plan image en un point quelconque $M$ (Fig. 7). Nous noterons par $\mathrm{P}$ l'intersection de la droite $\mathrm{O}_{3} \mathrm{M}$ avec la frontière $\mathrm{C}_{3}$ de la zone $S_{3}$. Tous les rayons réfléchis principaux d'angle $\varphi$ croiseront le plan image sur la droite $\mathrm{O}_{3} \mathrm{P}$. Le rayon réfléchi principal qui contient le point $\mathrm{P}$ correspond à l'angle maximum $\theta_{\mathrm{M}}$. Il est évident que généralement l'angle $\theta_{M}$ dépend de l'angle $\varphi$ :

$$
\theta_{\mathbf{M}}=\theta_{\mathbf{M}}(\varphi)
$$

Alors, le domaine de variation $[D]_{\mathrm{T}}$ sera le suivant :

a) $\varphi$ varie dans l'intervalle $[0,2 \pi]$.

b) $\theta$ varie dans l'intervalle $\left[0, \theta_{\mathbf{M}}(\varphi)\right]$.

La constante de normalisation $C$ sera donc déterminée par l'expression :

$$
C=\left[\int_{0}^{2 \pi} \mathrm{d} \varphi \int_{0}^{\theta_{\mathrm{M}}(\varphi)}|\sin \theta| \mathrm{d} \theta\right]^{-1} .
$$

L'erreur angulaire moyennée de $\theta,\langle\theta(\varphi)\rangle$, est déterminée par la relation usuelle :

$$
\langle\theta(\varphi)\rangle=\int_{0}^{\theta_{M}(\varphi)} \theta p(\theta) \mathrm{d} \theta .
$$

En appliquant les relations (16) et (17) au cas d'une zone d'imprécision de forme circulaire de rayon $r_{3}\left(\operatorname{tg} \theta_{\mathrm{M}}=r_{3} / T\right.$ où $\left.T=\left|\mathrm{O}_{1} \mathrm{O}_{3}\right|\right)$ on obtient la probabilité qu'un rayon réfléchi principal ait une déviation $\theta$ par rapport à l'axe optique :

$$
p(\theta)=\left[2 \pi\left(1-\cos \operatorname{arctg} \frac{r_{3}}{T}\right)\right]^{-1}|\sin \theta| .
$$

En utilisant la relation (20) on peut aussi obtenir :

$$
\langle\theta\rangle=\frac{\sin \operatorname{arctg} \frac{r_{3}}{T}-\operatorname{arctg} \frac{r_{3}}{T} \operatorname{cosarctg} \frac{r_{3}}{T}}{2 \pi\left(1-\operatorname{cosarctg} \frac{r_{3}}{T}\right)} .
$$

Nous établirons par la suite l'expression de la distribution des erreurs d'orientation, au niveau du plan image.

Dans ce but, on observe, en utilisant la formule (12) et la figure 7, qu'entre les paramètres $(a, b)$ et les coordonnées $(\hat{x}, \hat{y})$ du point où le rayon réfléchi principal rencontre le plan image existent les relations :

$$
a=\frac{\hat{x}}{T} \quad b=\frac{\hat{y}}{T} .
$$

Le jacobien des transformations (21) est :

$$
\frac{D(a, b)}{D(\hat{x}, \hat{y})}=T^{-2} .
$$


En remplaçant (11), (21), (22) en (8) nous trouvons :

$$
P(\mathbf{S})=C \iint_{[\mathrm{A}]}\left[\left(\frac{\hat{x}}{T}\right)^{2}+\left(\frac{\hat{y}}{T}\right)^{2}+1\right]^{-\frac{3}{2}} \mathrm{~d}\left(\frac{\hat{x}}{T}\right) \mathrm{d}\left(\frac{\hat{y}}{T}\right)
$$

l'intégration étant faite dans un sous-domaine [A] de la zone d'imprécision $S_{3}$. Il en résulte que la densité de probabilité $p(\hat{x}, \hat{y})$, avec le sens de probabilité que le rayon réfléchi principal rencontre le plan image au point $\mathbf{M}(\hat{x}, \hat{y})$ aura l'expression :

$$
p(\hat{x}, \hat{y})=C\left[\left(\frac{\hat{x}}{T}\right)^{2}+\left(\frac{\hat{y}}{T}\right)^{2}+1\right]^{-\frac{3}{2}} .
$$

La constante de normalisation $C$ est déterminée par la relation :

$$
\begin{array}{r}
C=\left\{\iint_{\mathrm{S}_{3}}\left[\left(\frac{\hat{x}}{T}\right)^{2}+\left(\frac{\hat{y}}{T}\right)^{2}+1\right]^{-\frac{3}{2}} \times\right. \\
\left.\times \mathrm{d}\left(\frac{\hat{x}}{T}\right) \mathrm{d}\left(\frac{\hat{y}}{T}\right)\right\}^{-1} .
\end{array}
$$

Nous noterons par $e$ la distance entre le point $\mathrm{M}$ et le centre optique $\mathrm{O}_{3}$. On voit que :

$$
e=\left(\hat{x}^{2}+\hat{y}^{2}\right)^{1 / 2} .
$$

A l'aide de l'expression (26) on trouve l'expression de l'erreur moyenne $\langle e\rangle$ :

$$
\langle e\rangle=\iint_{\mathrm{S}_{3}}\left(\hat{x}^{2}+\hat{y}^{2}\right)^{1 / 2} p(\hat{x}, \hat{y}) \mathrm{d}\left(\frac{\hat{x}}{T}\right) \mathrm{d}\left(\frac{\hat{y}}{T}\right) .
$$

Au cas d'une zone d'imprécision circulaire de rayon $r_{3}$ la constance $C \operatorname{de}(24)$ a la valeur :

$$
C=\frac{1}{2 \pi}\left\{1-\left[\left(\frac{r_{3}}{T}\right)^{2}+1\right]^{-1 / 2}\right\}^{-1} .
$$

3.2 L'ERREUR D'ORIENTATION DES HÉLIOSTATS POUR LESQUELS LE CENTRE $\mathrm{O}_{1}^{*}$ EST MOBILE. - Aucun des modèles cités, de calcul de l'intensité de la radiation solaire concentrée, ne prend explicitement en considération la possibilité que le centre de la surface réfléchissante soit mobile pendant le fonctionnement quotidien. Par la suite, nous traiterons cette possibilité, en exprimant les résultats sous une forme qui les rendent directement utilisables pour les techniques de calcul de ces modèles-là. Nous supposerons que la zone $S_{1}$, ainsi que les zones $S_{1}^{*}$ et $S_{3}^{*}$ déduites, ont une forme quelconque. Dans le cas général traité, la densité de probabilité est :

$$
p(a, b, u, v)=C\left(a^{2}+b^{2}+1\right)^{-2} .
$$

Pour donner un sens physique précis à la relation (28), nous remplacerons les paramètres $a, b$, par les paramètres $\hat{x}, \hat{y}$ de la zone d'imprécision. Conformément à la figure 8 nous avons :

$$
\begin{aligned}
& \hat{x}=u+T \operatorname{tg} \theta \cos \varphi \\
& \hat{y}=v+T \operatorname{tg} \theta \sin \varphi .
\end{aligned}
$$

Tenant compte de (12), les relations (29) deviennent :

$$
a=\frac{\hat{x}-u}{T} \quad b=\frac{\hat{y}-v}{T} .
$$

De plus, nous tiendrons compte que les transformations (30) n'opèrent pas sur les variables $u$ et $v$. Donc :

$$
\frac{u}{T}=\frac{u}{T} ; \quad \frac{v}{T}=\frac{v}{T} .
$$

Le jacobien des transformations (30) et (31) est :

$$
\frac{D(a, b, u / T, v / T)}{D(\hat{x} / T, \hat{y} / T, u / T, v / T)}=1 .
$$

Remplaçant (5), (30), (31), (32) en (8) nous trouvons :

$$
\begin{aligned}
& P=C \times \times \iiint \int_{\left[\mathrm{A}_{1}\right] \cup\left[\mathrm{A}_{3}\right]} \\
& \times {\left[\left(\frac{\hat{x}-u}{T}\right)^{2}+\left(\frac{\hat{y}-v}{T}\right)^{2}+1\right]^{-2} } \\
& \times \mathrm{d}\left(\frac{\hat{x}}{T}\right) \mathrm{d}\left(\frac{\hat{y}}{T}\right) \mathrm{d}\left(\frac{u}{T}\right) \mathrm{d}\left(\frac{v}{T}\right)
\end{aligned}
$$

$\left[A_{1}\right],\left[A_{3}\right]$ étant des sous-ensembles de $S_{1}^{*}$, et $S_{3}^{*}$ respectivement. La densité de probabilité correspondante à la probabilité (33) sera :

$$
p^{*}(u, v, \hat{x}, \hat{y})=C\left[\left(\frac{\hat{x}-u}{T}\right)^{2}+\left(\frac{\hat{y}-v}{T}\right)^{2}+1\right]^{-2}
$$

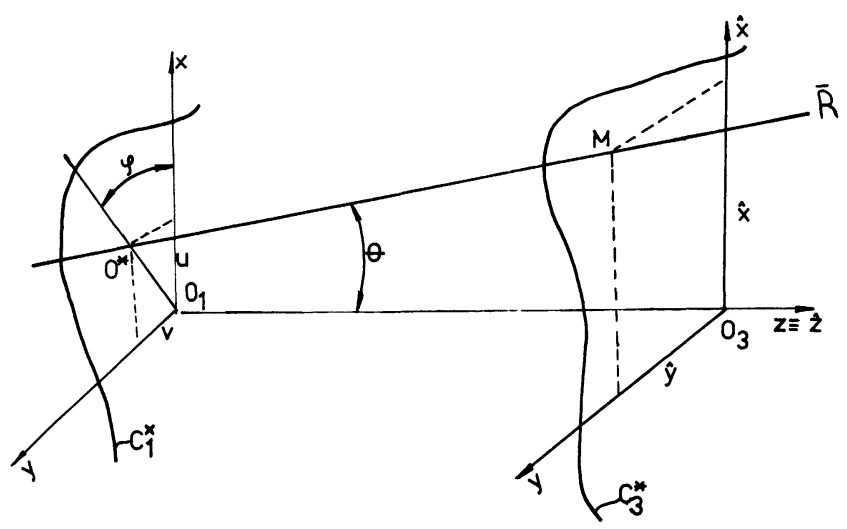

Fig. 8. - Les paramètres impliqués dans le calcul de la distribution des erreurs d'orientation (cas $\mathrm{O}_{1}^{*}$ mobile).

[The parameters involved in the calculus of the steering errors distribution (the case $\mathrm{O}_{1}^{*} \not \equiv \mathrm{O}_{1}$ ).] 
La constante de normalisation est déterminée par :

$$
\iiint \int_{\mathrm{s}_{1}^{*} \cup S_{3}^{*}} p^{*} \mathrm{~d}\left(\frac{\hat{x}}{T}\right) \mathrm{d}\left(\frac{\hat{y}}{T}\right) \mathrm{d}\left(\frac{u}{T}\right) \mathrm{d}\left(\frac{v}{T}\right) .
$$

Dans la pratique on s'intéresse surtout à la distribution :

$$
\hat{p}(\hat{x}, \hat{y})=\iint_{\mathrm{s}_{\mathbf{1}}^{*}} p^{*}(\hat{x}, \hat{y}, u, v) \mathrm{d}\left(\frac{u}{T}\right) \mathrm{d}\left(\frac{v}{T}\right)
$$

qui donne la probabilité pour que le rayon réfléchi principal ayant l'origine en $S_{1}^{*}$ croise le plan image au point $(\hat{x}, \hat{y})$. La valeur moyenne de l'erreur d'orientation est :

$$
\langle e\rangle=\iint_{\mathbf{s}_{3}^{*}}\left(\hat{x}^{2}+\hat{y}^{2}\right)^{1 / 2} \hat{p}(\hat{x}, \hat{y}) \mathrm{d}\left(\frac{\hat{x}}{T}\right) \mathrm{d}\left(\frac{\hat{y}}{T}\right) .
$$

L'existence des paramètres aléatoires supplémentaires $(u, v)$ fait que le rayon réfléchi principal ne puisse pas être caractérisé complètement par les angles $(\theta, \varphi)$. En effet, des droites ayant la même inclinaison par rapport à l'axe optique peuvent se différencier par des points $(u, v)$ différents. Pour pouvoir utiliser nos résultats, pour les modèles de calcul de la radiation solaire concentrée qui n'admettent que la possibilité que le centre $\mathrm{O}_{1}^{*}$ du miroir soit fixe, il est nécessaire qu'un rayon réfléchi principal puisse être caractérisé seulement par les deux angles d'inclinaison par rapport à l'axe optique. A cette fin nous introduisons dans le calcul deux angles fictifs, $\alpha$ et $\beta$, qui caractérisent un rayon réfléchi fictif $\bar{R}_{\mathrm{F}}$, ayant l'origine au point fixe $\mathrm{O}_{1}$. Un point quelconque $\mathbf{M}(\hat{x}, \hat{y})$ du plan image est complètement déterminé à l'aide des angles $\alpha$ et $\beta$ (Fig. 9).

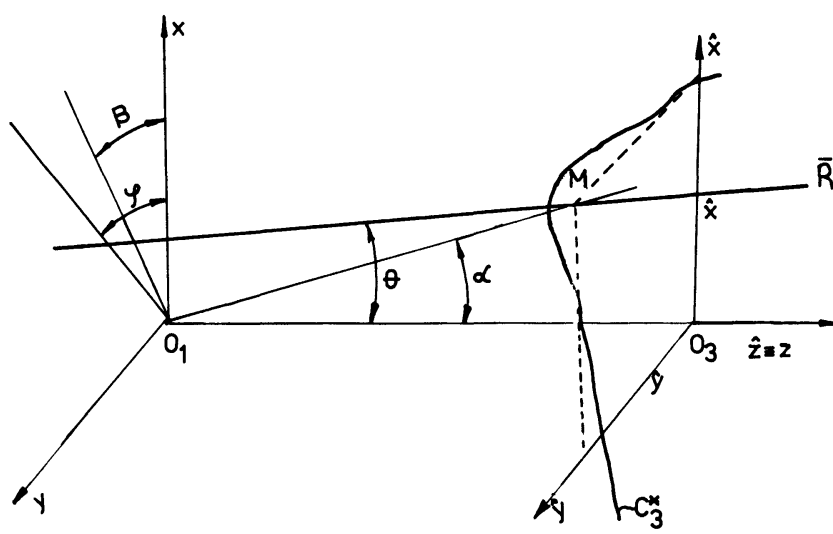

Fig. 9. - Les paramètres impliqués dans l'équivalence d'un rayon réfléchi principal quelconque $\bar{R}$ avec un rayon réfléchi principal fictif $O_{1} M$, ayant l'origine dans le point $O_{1}$. $(\varphi, \theta),(\alpha, \beta)$ caractérisent l'inclinaison de $\bar{R}$ et de $\mathrm{O}_{1} \mathrm{M}$, par rapport à $\mathrm{O}_{1} \mathrm{O}_{3}$ respectivement.

[The parameters involved in the equivalence of an arbitrary main reflected ray $\bar{R}$ with a fictitious main reflected ray $O_{1} M$, with the origin in the point $O_{1},(\varphi, \theta),(\alpha, \beta)$ characterize the inclination of the rays $\bar{R}$ and respectively $\mathrm{O}_{1} \mathrm{M}$, in comparison with $\mathrm{O}_{1} \mathrm{O}_{3}$.]
Revenons à l'expression (36), qui donne la probabilité $\hat{p}(\hat{x}, \hat{y})$ que l'ensemble des rayons réfléchis principaux $(Q)$, qui ont l'origine dans la zone $S_{1}^{*}$, passent par le point $\mathbf{M}(\hat{x}, \hat{y})$ du plan image. Par construction, le rayon réfléchi principal fictif $\bar{R}_{\mathrm{F}}$, rencontre le plan image au même point $\mathbf{M}(\hat{x}, \hat{y})$, tout comme l'ensemble $(\mathrm{Q})$ des rayons réfléchis principaux. Cela confère à la correspondance entre le rayon réfléchi $\bar{R}_{\mathrm{F}}$ et l'ensemble $(\mathrm{Q})$ un caractère biunivoque. Comme résultat, on peut toujours correspondre au rayon réfléchi principal fictif $\bar{R}_{\mathrm{F}}$ le nombre $\hat{p}(\hat{x}, \hat{y})$, spécifique pour l'ensemble $(\mathrm{Q})$. A l'aide de cette technique nous pourrons étudier par la suite les propriétés de l'ensemble $(Q)$, en utilisant le rayon réfléchi principal fictif $\bar{R}_{\mathrm{F}}$. On a :

$$
\begin{aligned}
& \hat{x}=T \operatorname{tg} \alpha \cos \beta \\
& \hat{y}=T \operatorname{tg} \alpha \sin \beta .
\end{aligned}
$$

La transformation (38) a le jacobien :

$$
\frac{D(\hat{x} / T, \hat{y} / T)}{D(\alpha, \beta)}=-\frac{\sin \alpha}{\cos ^{3} \alpha} \text {. }
$$

Remplaçant (30), (38), (39) en (8) on obtient :

$$
P_{\mathbf{F}}(\mathbf{S})=C \iint_{\left[\mathrm{A}_{3}\right]_{\mathbf{T}}} \hat{p}(\alpha, \beta)\left|\frac{\sin \alpha}{\cos ^{3} \alpha}\right| \mathrm{d} \alpha \mathrm{d} \beta
$$

où l'indice $\mathrm{F}$ signifie qu'il s'agit d'un ensemble $(\mathbf{S})$ des rayons réfléchis fictifs; $\hat{p}(\alpha, \beta)$ s'obtient en remplaçant $(\hat{x}, \hat{y})$ donné par (38) dans l'expression (36); $\left[\mathrm{A}_{3}\right]_{\mathrm{T}}$ est le sous-ensemble $\left[\mathrm{A}_{3}\right]$ de $\mathrm{S}_{3}^{*}$, soumis aux transformations (38). Il en résulte que la densité de probabilité sera :

$$
p_{\mathrm{F}}(\alpha, \beta)=C\left|\frac{\sin \alpha}{\cos ^{3} \alpha}\right| \hat{p}(\alpha, \beta) .
$$

La constante de normalisation sera déterminée par la condition :

$$
\iint_{\mathrm{S}_{3 \mathrm{~T}}} p_{\mathrm{F}}(\alpha, \beta) \mathrm{d} \alpha \mathrm{d} \beta=1
$$

où $S_{3 \mathrm{~T}}^{*}$ est le domaine $S_{3}^{*}$ soumis aux transformations (38). La distribution "fictive " $p_{\mathrm{F}}(\alpha, \beta)$ peut être utilisée dans les techniques de calcul de l'intensité de la radiation solaire concentrée qui prennent en considération les erreurs angulaires de réflexion rapportées à l'axe optique, dans l'hypothèse d'un centre $\mathrm{O}_{1}^{*}$ fixe. Par conséquent, les calculs effectués avec $p(\theta, \varphi)$ peuvent être répétés avec la distribution $p_{\mathrm{F}}(\alpha, \beta)$.

3.3 Le CAS Des SENSEURS NON CENTRÉs. - Nous considérons d'abord que le centre $\mathrm{O}_{2}^{*}$ de la zone insensible réduite ne coïncide pas avec le point $\mathrm{O}_{2}$, mais que la distance entre ces points est caractérisée par un vecteur de direction et de module bien déter- 
minés. Nous noterons par $\mathrm{O}_{3}^{\prime}$ l'intersection de l'axe $\mathrm{O}_{1} \mathrm{O}_{2}^{*}$ avec le plan image (Fig. 10). Conformément à la supposition antérieure, la distance $\mathrm{O}_{3} \mathrm{O}_{3}^{\prime}$ est bien déterminée et sera notée par $h$. Nous noterons les composantes du vecteur $\mathbf{h}$ avec $\left(h_{x}, h_{y}\right)$. Dans ce cas, l'erreur e (de composantes $e_{x}, e_{y}$ ) par rapport au centre optique, sera donnée par l'expression :

$$
\left(e_{x}, e_{y}\right)=(\hat{x}, \hat{y})+\left(h_{x}, h_{y}\right) .
$$

La zone d'imprécision déplacera son centre de $\mathrm{O}_{3}$ en $\mathrm{O}_{3}^{\prime}$, comme résultat d'une translation de vecteur $\mathbf{h}$. Après la translation, l'équation de la frontière $\mathrm{C}_{3}^{*}$ :

$$
\left(\mathrm{C}_{3}^{*}\right) \quad f(\hat{x}, \hat{y})=0
$$

aura l'expression correspondante à la frontière $C_{3}^{\prime}$ (voir Annexe) :

$$
\left(\mathrm{C}_{3}^{\prime}\right) \quad f\left(e_{x}, e_{y}\right)=0 \text {. }
$$

La densité de probabilité $\hat{p}(\hat{x}, \hat{y})$ se transformera en densité de probabilité $\hat{p}\left(e_{x}, e_{y}\right)$, gardant la même forme, mais changeant d'argument, selon la relation (43). Les calculs effectués en $\S 3.2$ avec la fonction $\hat{p}(\hat{x}, \hat{y})$ peuvent être répétés pour la fonction $\hat{p}\left(e_{x}, e_{y}\right)$.

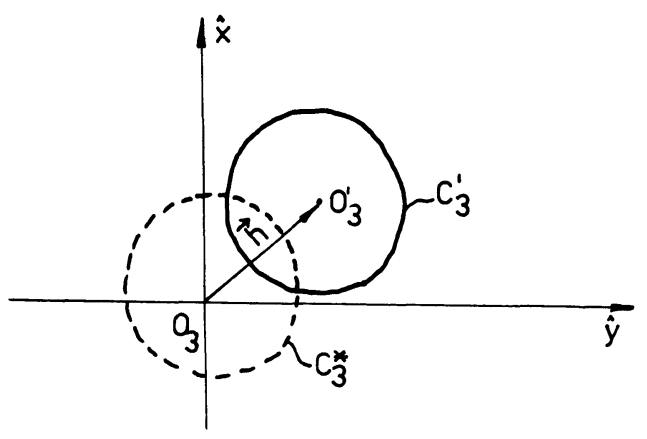

Fig. 10. - Construction de la courbe d'imprécision $\mathrm{C}_{3}^{\prime}$ d'un senseur dévié dans une position connue, par rapport à l'axe optique.

[The construction of the unprecise curve $\mathrm{C}_{3}^{\prime}$ of a sensory system swerved in a known position in comparison with the optic axis.]

Nous avons montré en $\S 2.3$ que, dans le cas général, à cause des erreurs de localisation et de réglage, le centre $\mathrm{O}_{2}^{*}$ de la zone insensible réduite n'a pas une position bien déterminée. Nous supposerons qu'il est distribué avec une certaine probabilité, dans une région $\mathrm{S}_{4}$, confinée par une courbe $\mathrm{C}_{4}$. La région $\mathrm{S}_{4}$, projetée du point $\mathrm{O}_{1}$, détermine au niveau du plan image une région $S_{5}$, confinée par une courbe $C_{5}$ (Fig. 11). Le point $\mathrm{O}_{3}^{*}$, l'intersection de la droite $\mathrm{O}_{1} \mathrm{O}_{2}^{*}$ avec le plan image, aura une probabilité $\tilde{p}(\hat{x}, \hat{y})$ de coïncider avec le point $(\hat{x}, \hat{y})$. Vu la nature probabiliste du déplacement du senseur par rapport à l'axe optique, la zone d'imprécision $\mathrm{S}_{3}^{*}$, déterminée antérieurement, s'étendra de plus dans le plan image.

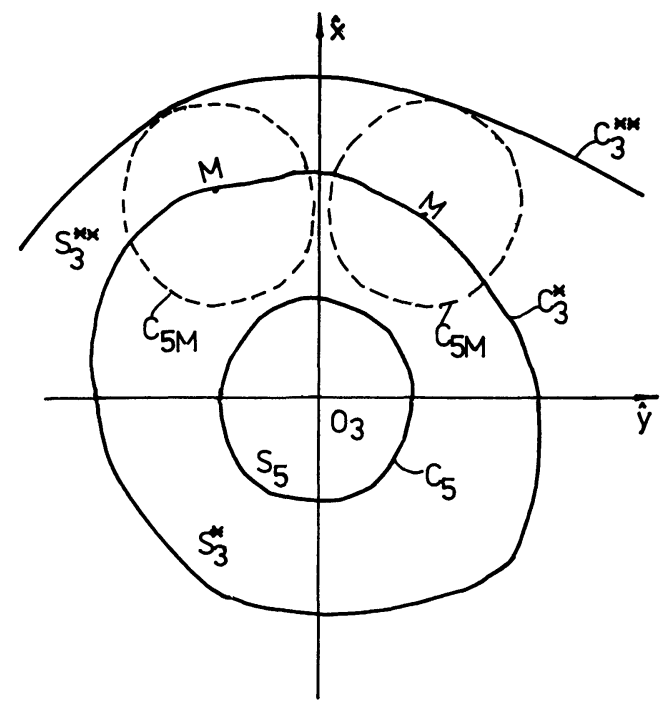

Fig. 11. - Construction de la courbe d'imprécision $\mathrm{C}_{3}^{* *}$ d'un senseur avec une déviation distribuée, par rapport à l'axe optique.

[The construction of the unprecise curve $\mathrm{C}_{3}^{* *}$ of a sensory system swerved in a distributed position in comparison with the optic axis.]

La construction de la nouvelle zone d'imprécision est la suivante (voir Annexe) :

a) La région $\mathrm{S}_{5}$ se translate, de sorte que le point qui coïncidait avec le point $\mathrm{O}_{3}$, se trouve dans un point $M$ quelconque de la courbe $C_{3}^{*}$. A la suite de la translation, la courbe $\mathrm{C}_{5}$ deviendra la courbe $\mathrm{C}_{5 \mathrm{M}}$.

$b$ ) L'opération $a$ ) se répète, pour tous les points $\mathrm{M}$ de la courbe $\mathrm{C}_{3}$. On obtiendra un ensemble de courbes $\left\{\mathrm{C}_{5 \mathrm{M}}\right\}$ (Fig. 11).

c) L'enveloppe $\mathrm{C}_{3}^{* *}$ des courbes $\left\{\mathrm{C}_{5 \mathrm{M}}\right\}$ est la frontière de la nouvelle zone d'imprécision, $S_{3}^{* *}$.

La probabilité $\breve{p}(\hat{x}, \hat{y})$ que le rayon réfléchi principal rencontre en un point quelconque $(\hat{x}, \hat{y})$ la zone d'imprécision $\mathrm{S}_{3}^{* *}$ est donnée par le produit de convolution :

$$
\breve{p}=\hat{p} * \tilde{p} .
$$

Développé, ce produit a l'expression :

$$
\breve{p}(\hat{x}, \hat{y})=\iint_{\left[S^{* *}\right]_{1 / T}} \hat{p}\left(\frac{\hat{x}}{T}-\mu, \frac{\hat{y}}{T}-v\right) \tilde{p}(\mu, v) \mathrm{d} \mu \mathrm{d} v
$$

où $\left[\mathrm{S}^{* *}\right]_{1 / \mathrm{T}}$ est le domaine de définition des variables $(\mu, v)$, résultant du domaine de variation $S_{3}^{* *}$ des paramètres $(\hat{x}, \hat{y})$, par multiplication avec $1 / T$. Les calculs effectués en $\S 3.2$ avec la fonction $\hat{p}(\hat{x}, \hat{y})$ peuvent se répéter avec la fonction $\breve{p}(\hat{x}, \hat{y})$.

$\mathrm{Si}$ la zone $S_{1}^{*}$ peut être considérée négligeable, dans le calcul de $\breve{p}$ on utilisera la fonction $p(\hat{x}, \hat{y})$ à la place de $\hat{p}(\hat{x}, \hat{y})$. 
Au cas où la zone d'imprécision $\mathrm{S}_{3}^{* *}$ et la distribution $\breve{p}$ ont un caractère sensiblement asymétrique par rapport au centre optique il est important de déterminer les coordonnées du point $\mathrm{N}(\langle\hat{x}\rangle,\langle\hat{y}\rangle)$ qui désignent l'erreur moyenne au niveau du plan image. Ces coordonnées sont déterminées par les relations usuelles :

$$
\begin{aligned}
& \langle\hat{x}\rangle=\iint_{\mathrm{S}_{3}^{* *}} \hat{x} \bar{p}(\hat{x}, \hat{y}) \mathrm{d}\left(\frac{\hat{x}}{T}\right) \mathrm{d}\left(\frac{\hat{y}}{T}\right) \\
& \langle\hat{y}\rangle=\iint_{\mathrm{S}_{3^{*}}^{*}} \hat{y} \breve{p}(\hat{x}, \hat{y}) \mathrm{d}\left(\frac{\hat{x}}{T}\right) \mathrm{d}\left(\frac{\hat{y}}{T}\right) .
\end{aligned}
$$

La connaissance du point $\mathrm{N}(\langle\hat{x}\rangle,\langle\hat{y}\rangle)$ nous donne l'occasion d'observer qu'il y a une possibilité pratique de diminuer l'erreur moyenne d'orientation. Cela consiste à déplacer le senseur de sorte que la la droite $\mathrm{O}_{1} \mathrm{O}_{2}^{*}$ croise le plan image en un point $\mathrm{O}_{3}^{\prime}$ de coordonnées $(-\langle\hat{x}\rangle,-\langle\hat{y}\rangle)$. Par cette translation le point $\mathrm{N}$ est superposé au point $\mathrm{O}_{3}$, ce qui entraîne que les erreurs d'orientation sont distribuées plus uniformément autour du centre optique.

3.4 LA VARIATION QUOTIDIENNE DES ERREURS D'ORIENTATION. - L'analyse du fonctionnement quotidien de l'héliostat nous permet d'obtenir des informations supplémentaires concernant les erreurs d'orientation. Supposons le cas où le centre de la surface réfléchissante $\mathrm{O}_{1}^{*}$ est fixe et le centre optique $\mathrm{O}_{3}$ se trouve au sud de l'héliostat.

Pour réfléchir les rayons incidents dans la direction de l'axe optique, la normale au point $\mathrm{O}_{1}^{*}$ à la surface réfléchissante tourne tout le long du matin, de l'est à l'ouest, et du nadir vers le zénith. En suivant la loi de la réflexion, le rayon réfléchi principal se déplacera d'ouest en est et du zénith au nadir.

De ce fait, le déclenchement de la correction d'orientation, se produira à l'intersection du rayon réfléchi principal avec l'arc $\overparen{E N}$ de la frontière $C_{3}$ (Fig. 12). Par conséquent, il y a de fortes chances que, dans la matinée, le rayon réfléchi principal rencontre la zone d'imprécision plus longtemps dans le secteur $\mathrm{EO}_{3} \mathrm{~N}$.

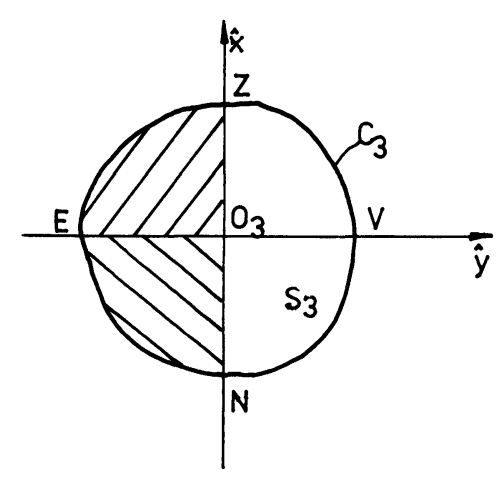

Fig. 12. - Zone hachurée $=$ zone restreinte d'imprécision.

[The restricted unprecise zone $\mathrm{O}_{3} \mathrm{ZEN}$.]
Pendant l'après-midi, la rotation de la même normale se fera d'est en ouest et du zénith au nadir et le rayon réfléchi principal rencontrera la zone d'imprécision de préférence dans le secteur $\mathrm{EO}_{3} \mathrm{Z}$. Si le centre optique se trouve au nord par rapport au miroir, une analyse similaire concluera que, le matin la zone d'imprécision sera restreinte particulièrement au secteur $\mathrm{EO}_{3} \mathrm{Z}$, et dans l'après-midi, au secteur $\mathrm{EO}_{3} \mathrm{~N}$.

Pour nous rapporter au fonctionnement des héliostats au cours d'une journée entière, on observe que, quelle que soit leur position par rapport au point $\mathrm{O}_{3}$, la zone d'imprécision se réduit particulièrement (mais non exclusivement) à la région $\mathrm{O}_{3} \mathrm{ZEN}$, recevant un fort caractère asymétrique par rapport au centre optique.

Les observations qualitatives mentionnées ci-dessus en ce qui concerne l'asymétrie de la distribution des erreurs d'orientation dues au mouvement apparent du Soleil, exigent pourtant une confirmation par un calcul rigoureux. Nous ne nous proposons pas de présenter ici les bases de cette technique rigoureuse de calcul. Nous remarquerons seulement que les résultats des paragraphes précédents ont été obtenus dans l'hypothèse implicite d'une variation indépendante des paramètres aléatoires $(a, b, u, v)$. Dans le cas étudié du point de vue qualitatif ci-dessus on a supposé, en plus, l'existence d'une corrélation entre les paramètres $a$ et $b$, qui décrivent le degré d'inclinaison du rayon réfléchi principal par rapport à l'axe optique. Cette corrélation correspondait au rapport qui existe entre les composantes du mouvement apparent du Soleil, quand on décompose ce mouvement selon deux directions (est-ouest et zénith-nadir).

4. Le cas des autres systèmes de commande et contrôle de l'orientation. - Nous essayerons par la suite de répondre à la question suivante : dans quelle mesure les résultats obtenus maintiennent leur validité dans des situations différentes du cas étudié (récepteur fixe et orientation contrôlée par des senseurs). Nous citerons comme exemple, le cas où le récepteur est rigidement lié au miroir concentrateur, donc mobile pendant la journée, le cas où le système de commande est programmé, etc. Pour répondre à cette question, il est nécessaire de distinguer deux problèmes : (i) déterminer la forme de la distribution des erreurs d'orientation; (ii) déterminer la constante de normalisation de cette distribution.

La forme de la distribution des erreurs d'orientation est profondément liée à l'aspect de la fonction invariante intégrale, qui est déterminée à son tour par la nature du processus d'orientation. Il est facile d'observer que, quelle que soit la modification par rapport au cas étudié initialement, l'essence du processus d'orientation ne changera pas. En effet, pendant le déroulement de ce processus, les rayons réfléchis principaux, qui auront la même expression (1), continueront à se transformer en rayons réfléchis de la même forme. 
Cette situation a comme résultat le fait que la fonction invariante intégrale ainsi que les distributions des erreurs d'orientation maintiendrons les expressions (5) et (7), respectivement (34) et (24).

En ce qui concerne la manière de déterminer la constante de normalisation, elle sera toujours donnée par les intégrales (35) et (10). Mais des différences apparaîtront dans la modalité dont on détermine le domaine d'intégration, qui dépend spécialement des particularités du système d'orientation utilisé.

5. Observations concernant les distributions proposées dans la littérature pour l'étude des erreurs d'orientation. - En utilisalı les résultats obtenus en $\S 3.2$, on fera une brève discussion sur la validité de certaines distributions des erreurs d'orientation proposées dans la littérature.

Nous nous rapporterons tout premièrement aux distributions de type gaussien, qui sont les plus anciennes et ont été utilisées parmi d'autres par Umarov [8] et McFee [6]. L'analyse détaillée du déroulement du processus d'orientation a prouvé que l'utilisation de ce type de distribution n'est pas justifiée. En effet, on observe de suite qu'aucune fonction gaussienne ne vérifie les systèmes d'équations différentielles (4) ou (6), ce qui correspond à une impossibilité de maintenir invariante la forme (1) du rayon réfléchi principal. Ceci est incompatible avec l'essence du processus d'orientation.

Le problème des erreurs d'orientation est abordé d'une autre manière par Lipps et Walzel [10], dans un modèle de calcul de l'intensité de la radiation concentrée par un champ d'héliostats. Ils acceptent $a$ priori, en attendant que l'expérience décide, le fait que l'effet des erreurs d'orientation peut être englobé avec d'autres corrections, dans une seule expression. Ainsi, pour prendre en considération l'effet de l'assombrissement vers le bord du Soleil sur l'intensité de la radiation concentrée, on introduit la distribution de l'éclat du Soleil, sous la forme :

$S(\theta)=\left\{\begin{array}{l}I_{0}+I_{1} \sin ^{2} \theta+I_{2} \sin ^{4} \theta+I_{3} \sin ^{6} \theta \\ 0 \quad \theta \geqslant \theta_{\mathrm{L}}=0.004660 \mathrm{rad} .\end{array}\right.$

où $\theta$ est l'angle déterminé par le rayon réfléchi principal et l'axe optique; $I_{0}, I_{1}, I_{2}, I_{3}$ sont des coefficients établis d'une manière empirique. Les auteurs supposent que ces coefficients empiriques pourraient prendre éventuellement en considération les perturbations atmosphériques et les erreurs d'orientation. Notre analyse a établi que dans le cas particulier où le centre du miroir est fixe, l'expression de la forme (49) peut prendre en considération les erreurs d'orientation en introduisant un terme supplémentaire, proportionnel à $|\sin \theta|$. De plus, dans le cas où l'orientation des héliostats est contrôlée par des senseurs, notre analyse a permis la détermination théorique de la grandeur du coefficient de proportionnalité. Au cas où ces erreurs ne peuvent être intégrées dans la distribution (49) les auteurs utilisent une distribution spéciale $G(\theta)$, dont ils ne donnent aucune indication sur sa forme. Si la zone d'imprécision est circulaire, comme expression de cette distribution on peut utiliser la relation $\left(16^{\prime}\right)$. Cette relation montre que la distribution dépend d'une manière assez compliquée de la distance entre le centre optique et l'héliostat, par opposition à la distribution de l'éclat du Soleil au niveau du plan image, qui dépend seulement de l'inverse du carré de la distance [10]. C'est pourquoi, l'hypothèse de calcul acceptée en [10], concernant la constance sur la surface du champ d'héliostats, du produit de convolution entre ces deux distributions n'est pas justifiée sans une analyse détaillée des phénomènes.

D'une manière originale, Hughes [7] étudie le cas particulier du système de commande de type par tout ou rien, ainsi que l'influence des erreurs d'orientation du système sur le flux de la radiation concentrée. Il part de l'hypothèse que, pour une certaine période de temps, les erreurs d'orientation sont uniformément distribuées dans une zone du plan image. La zone de distribution uniforme consiste en un carré de côté $2 \mathrm{~A}$, centré au point $\mathrm{O}_{3}$, ayant les axes de symétrie parallèles aux deux axes de mouvement du système mécanique. En utilisant la condition usuelle de normalisation, en [7] on détermine l'expression de la distribution uniforme des erreurs :

$$
f(\hat{x}, \hat{y})=\frac{1}{4 A^{2}}=\text { Cte }
$$

Cependant, une densité de probabilité uniforme n'est pas compatible avec le processus physique d'orientation, qui consiste à maintenir invariante la forme (1) du rayon réfléchi principal. En effet, une telle distribution ne vérifie aucun des systèmes d'équations (4) ou (6). Pourtant, on constate que, au cas où $\hat{x} / T$ et $\hat{y} / T$ sont très petits, une distribution de la forme $(50)$ peut constituer une bonne approximation de la distribution correcte (24), spécifique pour les systèmes de concentration dont le centre des miroirs peut être considéré fixe pendant le fonctionnement.

6. Conclusions. - L'analyse détaillée du processus d'orientation nous a permis d'établir le fait que la nature de ce processus peut être exprimée du point de vue géométrique par le groupe des transformations orthogonales de l'espace physique $\mathbf{R}_{\mathbf{3}}$. En partant de ce résultat, nous avons déterminé les expressions de la distribution des erreurs d'orientation dans l'hypothèse d'un système de commande qui utilise des senseurs. Ces expressions ont été présentées sous des formes qui puissent être directement utilisées dans les modèles $[4,5,6,10]$ de calcul de l'intensité de la radiation solaire concentrée.

On a montré que par la décentralisation du senseur par rapport à l'axe optique on peut diminuer l'erreur moyenne d'orientation. Puis, nous avons montré que la forme des distributions obtenues a un caractère général, qui ne dépend pas de la nature du système de 
commande et contrôle, des actionneurs mécaniques et aussi du fait que le récepteur soit fixe ou mobile. En même temps, nous avons presenté la manière avec laquelle la nature du système de commande et contrôle influence la détermination de la constante de normalisation. Les observations faites au chapitre 5 montrent qu'il n'y a pas justification théorique satisfaisante pour toutes les distributions des erreurs d'orientation proposées jusqu'à présent dans la littérature.

\section{ANNEXE}

Montrons la modalité de construire les surfaces et les courbes qui interviennent dans le présent travail. Aussi on a seulement besoin de la connaissance des expressions analytiques des courbes $C_{1}$ et $C_{2}$, dans le référentiel $\mathrm{O}_{1} x y z$.

1. Construction de la zone d'imprécision $S_{3}^{*}$. On suppose connue l'expression de la courbe $\mathrm{C}_{2}$ :

$$
\left(\mathrm{C}_{2}\right) \begin{aligned}
& A\left(x_{2}, y_{2}, z_{2}\right)=0 \\
& B\left(x_{2}, y_{2}, z_{2}\right)=0 .
\end{aligned}
$$

L'équation d'une droite mobile $(\delta)$, qui passe par un point fixe $\mathbf{M}\left(x_{1}, y_{1}, z_{1}\right) \in \mathbf{C}_{1}$ est :

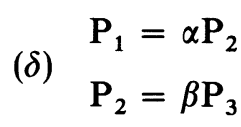

où $\alpha$ et $\beta$ sont des paramètres, et $\mathbf{P}_{1}, \mathbf{P}_{2}, \mathbf{P}_{3}$ sont des plans, d'équations :

( $\left.\mathbf{P}_{1}\right) a_{1}\left(x_{2}-x_{1}\right)+b_{1}\left(y_{2}-y_{1}\right)+c_{1}\left(z_{2}-z_{1}\right)=0$

( $\left.\mathrm{P}_{2}\right) \quad a_{2}\left(x_{2}-x_{1}\right)+b_{2}\left(y_{2}-y_{1}\right)+c_{2}\left(z_{2}-z_{1}\right)=0$

(P) $a_{3}\left(x_{2}-x_{1}\right)+b_{3}\left(y_{2}-y_{1}\right)+c_{3}\left(z_{2}-z_{1}\right)=0$.

En (A.3), $\left(x_{2}, y_{2}, z_{2}\right)$ sont les coordonnées d'un point quelconque $\mathrm{Q}$ de la droite $(\delta)$. Les coefficients $\left(a_{i}, b_{i}, c_{i}\right)$, $i=1,2,3$, sont des paramètres à fixer arbitrairement. La condition de non-parallélisme des plans $\mathbf{P}_{1}, \mathbf{P}_{2}, \mathbf{P}_{3}$ impose :

$$
\frac{a_{i}}{a_{j}} \neq \frac{b_{i}}{b_{j}} \neq \frac{c_{i}}{c_{j}}
$$

La condition que la droite $(\delta)$ rencontre la courbe $\mathrm{C}_{2}$ au point $\mathrm{Q}\left(x_{2}, y_{2}, z_{2}\right)$ est donnée par la solution des quatre équations (A.1) et (A.2), avec cinq inconnues, $\alpha, \beta, x_{2}, y_{2}, z_{2}$. Cette solution conduit à l'établissement d'un rapport entre les paramètres $\alpha$ et $\beta$ :

$$
K(\alpha, \beta)=0 .
$$

La relation (A.5) est la condition que la droite $(\delta)$ s'appuie sur la courbe $C_{2}$, déterminant un cône de sommet $\mathbf{M}\left(x_{1}, y_{1}, z_{1}\right)$. Remplaçant en (A.5) $\alpha$ et $\beta$ de
(A.2), fonction de $\left(x_{2}, y_{2}, z_{2}, x_{1} y_{1}, z_{1}\right)$ il en résulte :

$$
K\left(x_{2}, y_{2}, z_{2}, x_{1}, y_{1}, z_{1}\right)=0
$$

qui est l'équation du cône $\mathrm{K}_{\mathrm{M}}$, de sommet $\mathrm{M}\left(x_{1}, y_{1}, z_{1}\right)$ et de directrice la courbe $\mathrm{C}_{2}$. Supposons connue l'expression de la courbe $\mathrm{C}_{1}$ :

$$
\text { (C) } \begin{aligned}
& D\left(x_{1}, y_{1}, z_{1}\right)=0 \\
& F\left(x_{1}, y_{1}, z_{1}\right)=0 .
\end{aligned}
$$

Déterminer l'ensemble $\left\{K_{M}\right\}$ des cônes ayant le sommet sur la courbe $\mathrm{C}_{1}$ et comme directrice la courbe $C_{2}$, se ramène à résoudre les trois équations (A.6) et (A.7), avec six variables: $\left(x_{1}, y_{1}, z_{1}, x_{2}, y_{2}, z_{2}\right)$. Eliminons $y_{1}$ et $z_{1}$ de ce système, il résulte une expression de la forme :

$$
H\left(x_{2}, y_{2}, z_{2}, x_{1}\right)=0
$$

où $x_{1}$ apparaît comme paramètre. L'équation (A.8) est l'expression de l'ensemble $\left\{K_{M}\right\}$. Pour une valeur établie du paramètre $x_{1}$, nous aurons un cône particulier $\mathrm{K}_{\mathrm{M}}$. L'enveloppe de l'ensemble $\left\{\mathrm{K}_{\mathrm{M}}\right\}$ est donnée par la solution de l'équation (A.8) et de l'équation :

$$
\frac{\partial H}{\partial x_{1}}=J\left(x_{2}, y_{2}, z_{2}, x_{1}\right)=0 \text {. }
$$

En éliminant le paramètre $x_{1}$ des équations (A.8) et (A.9), nous arrivons à l'expression de l'enveloppe $E_{K}$ :

$$
\mathrm{E}_{\mathrm{K}}\left(x_{2}, y_{2}, z_{2}\right)=0 .
$$

La frontière $\mathrm{C}_{3}^{*}$ de la zone d'imprécision $\mathrm{S}_{3}^{*}$ résulte de l'intersection de l'enveloppe $\mathrm{E}_{\mathrm{K}}$ avec le plan $z_{2}=T$ :

$$
\left(\mathrm{C}_{3}^{*}\right) \begin{aligned}
& \mathrm{E}_{\mathrm{K}}\left(x_{2}, y_{2}, z_{2}\right)=0 \\
& z_{2}=T
\end{aligned}
$$

2. Construction de la courbe $\mathbf{C}_{3}$. - On construit le cône $K_{M}$ de sommet $O_{1}(0,0,0)$ :

$$
\mathrm{K}_{\mathbf{M}}\left(x_{2}, y_{2}, z_{2}, 0,0,0\right)=0 .
$$

La courbe $\mathrm{C}_{3}$ résulte de l'intersection du cône (A.12) avec le plan $z_{2}=T$.

3. Construction de la courbe $\mathrm{C}_{1}^{*} .-\mathrm{La}$ courbe $\mathrm{C}_{1}^{*}$ résulte de l'intersection de l'enveloppe $E_{K}$ avec le plan $z_{2}=0$.

4. Construction de la courbe $\mathbf{C}_{3}^{\prime}$ - - Supposons que, dans le système de coordonnées $\mathrm{O}_{3} \hat{x} \hat{y}$, la courbe $\mathrm{C}_{3}^{*}$ a pour expression :

$$
\left(\mathrm{C}_{3}^{*}\right) \quad L(\hat{x}, \hat{y})=0 .
$$

La courbe $C_{3}^{\prime}$ s’écrit donc (Fig. 10) :

$$
\left(C_{3}^{\prime}\right) L\left(\hat{x}-h_{x}, \hat{y}-h_{y}\right)=0 .
$$


5. Construction de la courbe $\mathbf{C}_{3}^{* *}$. - Supposons que l'équation de $\mathrm{C}_{3}^{*}$, dans le repère $\mathrm{O}_{3} \hat{x} \hat{y}$, soit :

$$
\left(\mathrm{C}_{3}^{*}\right) \quad L(\hat{x}, \hat{y})=0
$$

et

$$
\left(\mathrm{C}_{5}\right) \quad R(\hat{\hat{x}}, \hat{\hat{y}})=0
$$

dans le même repère.

On translate la courbe $C_{5}$ en un point $M$ quelconque de la courbe $\mathrm{C}_{3}^{*}$. L'équation de la courbe translatée $\mathrm{C}_{5 \mathrm{M}}$ sera :

$$
\left(C_{5 M}\right) \quad R(\hat{\hat{x}}-\hat{x}, \hat{\hat{y}}-\hat{y})=0 .
$$

On élimine $\hat{y}$ du système des équations $\left(\mathrm{A} .13^{\prime}\right)$ et
(A.16) et nous obtenons l'équation :

$$
V(\hat{\hat{x}}, \hat{\hat{y}}, \hat{x})=0
$$

qui représente l'ensemble $\left\{\mathrm{C}_{5 \mathrm{M}}\right\}$ des courbes $\mathrm{C}_{5 \mathrm{M}}$ translatées en des points différents de la courbe $C_{3}^{*}$. En (A.17), $\hat{x}$ est un paramètre et l'équation de l'enveloppe de l'ensemble $\left\{C_{5 M}\right\}$ sera donnée par (A.17) et par :

$$
\frac{\partial V}{\partial \hat{x}}=W(\hat{\hat{x}}, \hat{\hat{y}}, \hat{x})=0 .
$$

En éliminant de (A.17) et (A.18) le paramètre $\hat{x}$, on obtient l'équation de la courbe $\mathrm{C}_{3}^{* *}$ :

$$
\left(\mathrm{C}_{3}^{* *}\right) \quad U(\hat{\hat{x}}, \hat{\hat{y}})=0 \text {. }
$$

\section{Bibliographie}

[1] Edenburg, M. W., Solar Energy 18 (1976) 437.

[2] Strobel, G. L., Burkhard, D. G., Solar Energy 20 (1978) 25.

[3] Neville, R. C., Solar Energy 20 (1978) 7.

[4] Riaz, M., Gurr, T., Solar Energy 19 (1977) 185.

[5] LIPPS, F. W., Solar Energy 18 (1976) 555.

[6] McFee, R. H., Appl. Opt. 14 (1975) 1493.

[7] Hughes, R. O., Solar Energy 24 (1980) 83.

[8] Umarov, G. Y., Geliotekhnika 3 (1967) 5.

[9] Igel, E. A., Hughes, R. L., Solar Energy 22 (1979) 283.

[10] Lipps, F. W., Walzel, M. D., Solar Energy 21 (1978) 113.

[11] ITTNER III, W. B., Solar Energy 24 (1980) 221.

[12] Hughes, R. O., 13th IECEC, San Diego, California (August 1978).
[13] Hughes, R. O., ASME Winter Annual Meeting, Paper 78-WA/SOL-4, San Francisco, California (December 1978).

[14] BĂdeSCU, V., St. Cerc. Fiz. 33 (1981) 455.

[15] Trombe, F., Applications thermiques de l'énergie solaire dans le domaine de la recherche et de l'industrie (CNRS, Paris) 1961, p. 87-126.

[16] TouchaIs, M., L'énergie solaire et sa maîtrise industrielle, Fasc. 2 et 4 (Estrel) 1979.

[17] Kendall, M. G. K., Moran, P. A. P., Geometrical Probability (Charles Griffin and Company, London) 1963.

[18] Stoka, M., Theodorescu, R., Probabilitate si geometrie (Ed. Stiintifica, Bucuresti) 1966. 